\title{
Análise dos parâmetros fitossociológicos em fragmento de Floresta Estacional Subtropical
}

\author{
Alexsandra Cezimbra Quevedo $\oplus^{1^{*}}$ Maristela Machado Araujo $\odot^{1}$ Roberta Rodrigues Roubuste $\bigodot^{1}$ \\ Mateus Alves Saldanha $\mathbb{0}^{1}$ Suelen Carpenedo Aimi ${ }^{1}{ }^{1}$ Luéli Fabrine Stroher Ritter ${ }^{1}{ }^{1}$
}

${ }^{1}$ Universidade Federal de Santa Maria. Av. Roraima no 1000 Cidade Universitária, Camobi, CEP: 97105-900, Santa Maria, RS, Brasil

\begin{abstract}
Original Article
*Corresponding author: alexsandra99926919@hotma il.com

Keywords:

Stratum

Dispersion syndrome

Phytosociology

Palavras-chave:

Estrato

Síndrome de dispersão

Fitossociologia

Received in

2020/10/24

Accepted on

2021/03/02

RESUMO: A caracterização da vegetação e fragmentos florestais subsidia informações para o desenvolvimento regional, assim como o entendimento sobre a estrutura e dinâmica dos ecossistemas. Desse modo, objetivou-se caracterizar a estrutura florística e avaliar a dinâmica de um fragmento de Floresta Estacional Subtropical para a fauna regional. Para isso, foram utilizadas faixas paralelas dentro das quais foram distribuídas parcelas temporárias de $10 \mathrm{~m} \times 10 \mathrm{~m}$, equidistantes $20 \mathrm{~m}$, e todos os indivíduos com CAP (circunferência medida a 1,30 $\mathrm{m}$ da superfície do solo) $\geq 15 \mathrm{~cm}$ foram inventariados. Observou-se 29 espécies pertencentes a 16 famílias no estrato médio, no estrato inferior 11 espécies em 8 famílias e no estrato superior 15 espécies de 11 famílias. $\mathrm{O}$ fragmento apresentou mais de $70 \%$ de dispersão dos frutos por zoocoria, indicando sua importância para conservação da fauna regional. Ocotea puberula, Lithraea molleoides, Casearia sylvestris, Allophylus edulis, Cupania vernalis, Luehea divaricata, Actinostemon concolor, Piper aduncum e Aiouea saligna foram as espécies de maior relevância, apresentando valores de importância elevados. A forma de dispersão de frutos e sementes de cada espécie é fundamental para a manutenção da biodiversidade e regeneração natural, influenciando diretamente na colonização das espécies e no modo de distribuição. As espécies mais representativas apresentaram padrão de distribuição espacial agrupado, possivelmente por sua preferência a condições ambientais específicas. Esse estudo possibilitou salientar a importância ecológica das espécies em diferentes sinúsias e a dispersão dos frutos pela fauna, contribuindo para a regeneração natural e conservação da biodiversidade em fragmento de Floresta Estacional Subtropical.
\end{abstract}

Published in

$2021 / 06 / 30$

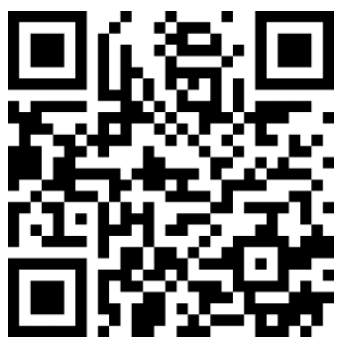

DOI:

http://dx.doi.org/10.34062/af s.v8i1.11343

\section{(cc) BY}

\section{Analysis of the phytosociological parameters in a fragment of Subtropical Seasonal Forest}

\begin{abstract}
The characterization of vegetation in forest fragments subsidizes information for regional development, as well as the understanding of ecosystem structure and dynamics. Thus, the aim was to characterize the floristic structure and evaluate the dynamics of a fragment of Subtropical Seasonal Forest for the regional fauna. For this, parallel strips were used within which temporary plots of $10 \mathrm{~m} \times 10 \mathrm{~m}$ were distributed, equidistant $20 \mathrm{~m}$, and all individuals with PAC (circumference measured at $1.30 \mathrm{~m}$ from the soil surface) $\geq 15 \mathrm{~cm}$ were inventoried. 29 species belonging to 16 families were observed in the middle layer, the lower layer 11 species in 8 families and the upper layer 15 species of 11 families. The fragment showed more than $70 \%$ fruit dispersion per zoocoria, indicating its importance for the conservation of regional fauna. Ocotea puberula, Lithraea molleoides, Casearia sylvestris, Allophylus edulis, Cupania vernalis, Luehea divaricata, Actinostemon concolor, Piper aduncum and Aiouea saligna were the most important species, showing high values of importance. The form of dispersion of fruits and seeds of each species is fundamental for the maintenance of biodiversity and natural regeneration, directly influencing the colonization of species and the mode of distribution. The most representative species presented a grouped pattern of spatial distribution, possibly due to their preference for specific environmental conditions. This study made it possible to highlight the ecological importance of the species in different sinusias and the dispersion of the fruits by the fauna, contributing to the natural regeneration and conservation of biodiversity in fragments of Subtropical Seasonal Forest.
\end{abstract}




\section{Introdução}

A região subtropical e tropical do planeta corresponde a $31 \%$ da superfície terrestre total, sendo coberta por florestas que representam, aproximadamente, mais de quatro bilhões de hectares (FAO, 2010), sendo parte deste valor, formado por Floresta Estacional. Entretanto, no Brasil, o Bioma Mata Atlântica, que contempla essa tipologia florestal apresenta sérios problemas de fragmentação, necessitando de planejamento da conservação da biodiversidade, a partir de ações que possibilitem a conectividade entre fragmentos, dentro de cada sub-região (RIBEIRO et al., 2009).

Em regiões com estações bem definidas, como no Sul do Brasil onde ocorre baixa temperatura, é comum a perda de folhas das árvores do dossel durante o inverno. Nessas florestas, também denominadas de Floresta Estacional Subtropical, durante a estação fria, cerca de $50 \%$ das árvores do estrato superior apresentam caducifólia (VACCARO et al., 2011).

Diante dessas variações climáticas, assim como topográfica e condições edafológicas, a Floresta Estacional Subtropical é complexa em sua ecologia, exibindo vegetação formada por mosaicos com evidentes variações fitofisionômicas, que acompanham gradientes ambientais, apresentando diferentes relações entre grupos de espécies e habitats (SCIPIONI et al., 2010; CHAMI et al., 2011; MARCUZZO et al., 2013).

Nesse contexto, a caracterização da vegetação dentro dos fragmentos subsidia informações importantes para o desenvolvimento regional. Esse conhecimento é capaz de facilitar a decisão de utilização dos recursos naturais (SOUZA et al., 2020).

O estudo da vegetação em áreas reduzidas busca identificar sua contribuição na conectividade florestal, assim como na necessidade de isolamento da área do acesso do gado, do enriquecimento com outras espécies regionais ou ações que contribuam para manutenção da diversidade regional. Além disso, visa mostrar uma estrutura e dinâmica sucessional diferente das observadas em áreas extensas, com formações florestais em estágios mais avançados de desenvolvimento (SCCOTI et al., 2011; CALLEGARO et al., 2012).

O entendimento sobre a estrutura e dinâmica dos ecossistemas, destacam-se com grande importância para auxiliar na quantificação do potencial de cada formação, sendo primordial para a utilização de forma racional das florestas nativas (CHAMI et al., 2011). A caracterização da estrutura de florestas naturais tornou-se foco de pesquisa com observação da estrutura vertical, da estrutura diamétrica e a estrutura horizontal (índice de valor de importância, dominância, densidade e frequência) de comunidades arbóreas (ARRUDA et al., 2011).
Nesse sentido, o objetivo do presente estudo foi caracterizar a distribuição espacial, estrutura florística e fitossociológica por estrato florestal, bem como avaliar a dispersão dos frutos e o tipo de dispersão de um fragmento de Floresta Estacional Subtropical para a fauna regional.

\section{Material e Métodos}

O estudo foi realizado no município de Santa Maria, região Central do Estado do Rio Grande do Sul, em um fragmento florestal, localizado nas coordenadas $29^{\circ} 47^{\prime} 40,35^{\prime}$ ' de latitude sul e $53^{\circ} 40^{\prime} 07,72^{\prime \prime}$ de longitude oeste, com área de cerca de 7,5 hectares e altitude de $110 \mathrm{~m}$.

O município de Santa Maria situa-se na zona de transição entre a Depressão Central e a escarpa arenito-basáltica do Planalto Meridional Brasileiro. A região pertence à Bacia Hidrográfica do VacacaíVacacaí Mirim e está localizada na porção centroocidental do Estado do Rio Grande do Sul (SEMA, 2020). De acordo com a classificação de Köppen, o clima da região é do tipo $\mathrm{Cfa}$, subtropical úmido, definido com temperatura média anual de $18,3{ }^{\circ} \mathrm{C}$ e precipitação média anual de $1.589,3 \mathrm{~mm}$, bem distribuída ao longo do ano (MATZENAUER et al., 2011).

A vegetação da área amostrada possui domínio fitogeográfico de Floresta Estacional Subtropical, pertencente ao Bioma Mata Atlântica. Nesse fragmento florestal, em alguns pontos da área florestal há o acesso do gado.

No estudo utilizou-se amostragem sistemática por meio de transecto (faixa), foram desmarcadas três faixas perpendiculares, cada uma com cinco parcelas temporárias $(10 \mathrm{~m} \times 10 \mathrm{~m})$. Para a análise da vegetação arbórea, todos os indivíduos com CAP (circunferência medida a $1,30 \mathrm{~m}$ da superfície do solo) $\geq 15 \mathrm{~cm}$ foram amostrados, sendo identificada a espécie, avaliada a altura estimada, posição sociológica e a medição do CAP.

Na otimização da amostragem utilizou-se a curva espécie-área. Os parâmetros fitossociológicos como densidade relativa (DR), frequência relativa (FR), dominância relativa (DoR) e índice de valor de importância (IVI) (\%) foram determinados para todas as espécies, e o índice de diversidade de Shannon (H') e equabilidade de Pielou (J') para a comunidade como um todo, conforme MullerDombois e Ellenberg (1974). Estes parâmetros foram calculados por meio do software FITOPAC 2.1 (SHEPHERD, 2010).

Além desses dados, cada indivíduo amostrado foi observado quanto a sua posição fitossociológica, considerando os três estratos: superior, médio e inferior. A avaliação dos estratos foi procedida de forma visual em: superior, correspondendo as árvores com copas emergentes que recebiam incidência de luz no topo e pelo menos 
uma das laterais; médio quando a luz incidia no topo da copa ou parcialmente no topo e na lateral; e inferior representado pelos indivíduos que ocorrem no sub-bosque.

De acordo com metodologia de Pinheiro et al. (2001), as espécies foram classificadas quanto à síndrome de dispersão por meio da morfologia dos frutos, sendo categorizadas em três grupos: anemocóricas (dispersão pelo vento), autocóricas (dispersão pela gravidade) e zoocóricas (dispersão pelos animais).
A distribuição espacial das dez espécies mais abundantes na área amostral foi calculada por meio do Índice de Morisita. Esse índice caracteriza-se pela independência da média e do número de amostras, sendo que quando $\mathrm{I}=1$, a distribuição é aleatória; $\mathrm{I}<$ 1, a distribuição é uniforme e I > 1 indica distribuição agrupada (NETO et al., 1976).

O cálculo do Índice de Morisita procedeu-se inicialmente com a elaboração da tabela para cada uma das dez espécies analisadas (Tabela 1).

Tabela 1. Representação para medida de dispersão por meio do Índice de Morisita para cada espécie.

\begin{tabular}{l|l|l|l}
\hline $\mathrm{x}$ & $\mathrm{f}(\mathrm{x})$ & $\mathrm{f}(\mathrm{x}) * \mathrm{x}$ & $\mathrm{f}(\mathrm{x}) * \mathrm{x}^{2}$ \\
\hline 0 & & & \\
\hline 1 & & & \\
\hline 2 & & & \\
\hline$:$ & & & \\
\hline $\mathrm{n}$ & & & \\
\hline$\Sigma$ & & & \\
\hline
\end{tabular}

Onde: $\mathrm{X}=$ número de indivíduos de 1 a n (número de indivíduos máximo que ocorre na parcela). $\mathrm{f}(\mathrm{x})=$ número de parcelas em que ocorre determinado número de indivíduos da espécie. $\sum=$ somatório.

Posteriormente, com os valores calculados na Tabela 1, utilizou-se a fórmula do Índice de Morisita (Equação 1), obtendo a distribuição espacial de cada espécie abundante na área amostral.

$$
I=\sum_{i}^{n} f(x) \times \frac{\sum_{i=1}^{n} f(x) \times x^{2}-\sum_{i=1}^{n} f(x) \times x}{\left(\sum_{i=1}^{n} f(x) \times x\right) \times\left[\left(\sum_{i=1}^{n} f(x) \times x\right)-1\right]}
$$

Em que: $\mathrm{I}$ = índice de Morisita; $\mathrm{x}$ = número de indivíduos de 1 a $\mathrm{n}$ (número de indivíduos máximo que ocorre na parcela); $\mathrm{f}(\mathrm{x})=$ número de parcelas em que ocorre determinado número de indivíduos da espécie; $\sum=$ somatório.

\section{Resultados e Discussão}

A dispersão dos frutos por zoocoria destacouse em $70 \%$ do fragmento florestal, e as espécies de maior representatividade apresentaram padrão de distribuição espacial agrupado (Tabela 2).

$\mathrm{Na}$ análise da estrutura vertical (Tabela 2), verificou-se que ocorreram o total de 35 espécies distribuídas em 20 famílias.

$\mathrm{Na}$ composição florestal do fragmento observou-se que as espécies com maior predominância foram: C. sylvestris, A. edulis, $L$. divaricata, O. puberula, C. vernalis, L. molleoides, A. concolor, P. aduncum e A. saligna (Tabela 3).

No estrato superior ocorreram 15 espécies distribuídas em 11 famílias, sendo que dentre estas as que apresentaram maior índice de valor de importância (IVI) foram O. puberula $(27,37 \%), L$. molleoides (18,47\%), C. sylvestris $(34,1 \%)$, A. edulis $(6,3 \%)$ e $C$. vernalis $(5,57 \%)$. No estrato superior, a família com maior riqueza florística foi a Lauraceae (três espécies), seguida de Myrtaceae e Sapindaceae (cada uma com duas espécies) e as demais somente com uma espécie. Essas espécies são normalmente encontradas em fragmentos de Floresta Estacional Subtropical (SCCOTI et al., 2011).

A maior riqueza florística da área amostrada foi observada no estrato médio (Tabela 3), com 29 espécies distribuídas em 16 famílias. As espécies com maiores IVI foram $C$. sylvestris $(18,27 \%), A$. edulis $(10,23 \%)$, L. divaricata $(7,97 \%)$, O. puberula $(7,17 \%)$ e $C$. vernalis $(5,8 \%)$. Entre essas espécies, $O$. puberula no estrato superior foi a espécie que apresentou o maior IVI, diferentemente no estrato médio, que ficou em quarta posição, quanto a este índice, mostrando que espécies que recebem incidência direta da luz, ou seja, no dossel florestal, apresentam maiores valores quanto aos parâmetros de DR, FR e DoR, influenciando diretamente no índice de valor de importância da espécie. 
Tabela 2. Nome científico, família e Síndrome de dispersão de cada espécie em fragmento de Floresta Estacional Subtropical.

Família

\begin{tabular}{|c|c|c|}
\hline Anacardiaceae & Lithraea molleoides Hieron/Na & Zoo \\
\hline Arecaceae & Syagrus romanzoffiana (Cham.) Glassman & Zoo \\
\hline Boraginaceae & Cordia americana (L.) Gottschiling \& J. S. Mill. /Bo & Ane \\
\hline Cannabaceae & Trema micrantha (L.) Blume & Auto \\
\hline Euphorbiaceae & Actinostemon concolor (Spreng.) Müll.Arg. & Auto \\
\hline Euphorbiaceae & Gymnanthes klotzschiana Müll.Arg. & Auto \\
\hline Fabaceae & Enterolobium contortisiliquum (Vell.) Morong & Auto \\
\hline Fabaceae & Machaerium paraguariense Hassi & Ane \\
\hline Lauraceae & Aiouea saligna Meisn/L & Zoo \\
\hline Lauraceae & Nectandra lanceolata Ness \& Mart. & Zoo \\
\hline Lauraceae & Nectandra megapotamica (Spreng.) Mez/ L & Zoo \\
\hline Lauraceae & Ocotea puberula (Meisn.) Mez/L & Zoo \\
\hline Lauraceae & Ocotea pulchella (Ness \& Mart.) Mez & Zoo \\
\hline Malvaceae & Luehea divaricata Mart. \& Zucc./ M & Zoo \\
\hline Malvaceae & Pavonia sepium A. St.-Hill & Zoo \\
\hline Meliaceae & Cabralea canjerana (Vell.) Mart. & Zoo \\
\hline Meliaceae & Trichilia clausseni C. DC. & Zoo \\
\hline Moraceae & Sorocea bonplandii W. C. Burger et al. & Auto \\
\hline Myrtaceae & Campomanesia xanthocarpa O. Berg & Zoo/Auto \\
\hline Myrtaceae & Eugenia rostrifolia D. Legrand & Zoo \\
\hline Myrtaceae & Eugenia uniflora L. & Zoo \\
\hline Myrtaceae & Psidium guajava L. & Zoo \\
\hline Piperaceae & Piper aduncum L. & Zoo \\
\hline Poligonaceae & Ruprechtia laxiflora C. DC. & Ane \\
\hline Primulaceae & Myrsine umbellata Mart. & Zoo \\
\hline Rubiaceae & Chomelia obtusa Cham. \& Schltdl./Ru & Zoo \\
\hline Rutaceae & Citrus sp. & Zoo \\
\hline Rutaceae & Zanthoxylum rhoifolium Engl. & Zoo \\
\hline Salicaceae & Casearia sylvestris $\mathrm{Sw} . / \mathrm{S} 1$ & Zoo \\
\hline Sapindaceae & Allophylus edulis A. St -Hil. A.Juss. \& Cambess.) Hieron. Ex Niederl./Sap & Zoo \\
\hline Sapindaceae & Cupania vernalis Cambess./Sap & Zoo \\
\hline Sapindaceae & Diatenopteryx sorbifolia Radlk. & Ane \\
\hline Sapindaceae & Matayba elaeagnoides Radlk. & Zoo \\
\hline Sapotaceae & Chrysophyllum marginatum (Hook. \& Arn.) Radlk. & Zoo \\
\hline Urticaceae & Urera sp. & Zoo \\
\hline
\end{tabular}

Nome Científico

SD

Onde: SD = síndrome de dispersão; Zoo = zoocórica; Ane= anemocórica. Auto = autocórica.

A família com maior riqueza florística no estrato médio foi a Lauraceae (com cinco espécies), seguida da família Myrtaceae e Sapindaceae (com quatro espécies, respectivamente) e o restante com duas ou apenas uma espécie.
O estrato inferior (Tabela 3) apresentou 11 espécies distribuídas em oito famílias. As espécies com maiores índices de valores de importância foram A. concolor $(37,47 \%)$, A. edulis $(19,17 \%), L$. molleoides (13,36\%), P. aduncum $(6,6 \%)$ e $A$. saligna $(3,75 \%)$. A família Sapindaceae foi a de 
Quevedo et al.

maior riqueza florística no estrato inferior, com a presença de três espécies, seguida de Rutaceae, com duas espécies. As demais famílias apresentaram apenas uma espécie.

Tabela 3. Parâmetros fitossociológicos dos estratos superior, médio e inferior (CAP $\geq 15 \mathrm{~cm}$ ), em fragmento de Floresta Estacional Subtropical no município de Santa Maria, Rio Grande do Sul.

\begin{tabular}{|c|c|c|c|c|c|c|c|c|c|c|c|c|c|c|c|}
\hline \multirow[b]{2}{*}{ Nome Científico } & \multicolumn{5}{|c|}{ Estrato superior } & \multicolumn{5}{|c|}{ Estrato médio } & \multicolumn{5}{|c|}{ Estrato inferior } \\
\hline & NI & DR & FR & Dor & $\begin{array}{l}\text { IVI } \\
(\%) \\
\end{array}$ & NI & DR & FR & Dor & $\begin{array}{l}\text { IVI } \\
(\%) \\
\end{array}$ & NI & DR & FR & Dor & $\begin{array}{l}\text { IVI } \\
(\%) \\
\end{array}$ \\
\hline A. concolor & - & - & _- & - & - & 3 & 3,57 & 3,57 & 0,9 & 2,68 & 17 & 48,6 & 48,6 & 30,5 & 37,47 \\
\hline A. saligna & 3 & 6,67 & 5,88 & 1,41 & 4,67 & 3 & 3,57 & 5,36 & 3,13 & 4,03 & 1 & 2,86 & 2,86 & 2,85 & 3,75 \\
\hline A. edulis & 3 & 6,67 & 8,82 & 3,36 & 6,3 & 10 & 11,9 & 10,7 & 8,04 & 10,23 & 6 & 17,1 & 17,1 & 23,7 & 19,17 \\
\hline C. canjerana & - & - & - & - & - & 2 & 2,38 & 3,57 & 2,91 & 2,95 & - & - & - & - & - \\
\hline C. xanthocarpa & 1 & 2,22 & 2,94 & 2,21 & 2,46 & 1 & 1,19 & 1,79 & 1,88 & 1,62 & _- & _ & _- & - & - \\
\hline C. sylvestris & 6 & 13,3 & 14,7 & 6,05 & 11,37 & 19 & 22,6 & 12,5 & 19,7 & 18,27 & 1 & 2,86 & 2,86 & 1,19 & 3,2 \\
\hline C. obtusa & 1 & 2,22 & 2,94 & 3,02 & 2,73 & _- & _- & _- & _- & _ & - & _- & _ & _- & _- \\
\hline C. marginatum & - & - & - & _ & - & 2 & 2,38 & 3,57 & 4,18 & 3,37 & - & - & _- & - & - \\
\hline Citrus sp. & - & - & - & - & - & - & - & - & - & - & 1 & 2,86 & 2,86 & 2,64 & 3,68 \\
\hline C. americana & 1 & 2,22 & 2,94 & 2,89 & 2,68 & - & - & - & - & - & - & - & - & - & - \\
\hline C. vernalis & 3 & 6,67 & 5,88 & 4,16 & 5,57 & 3 & 3,57 & 5,36 & 8,51 & 5,8 & 1 & 2,86 & 2,86 & 1,09 & 3,17 \\
\hline D. sorbifolia & - & _ & - & - & _- & 1 & 1,19 & 1,79 & 1,3 & 1,42 & - & _- & _ & _ & _ \\
\hline E. contortisiliquum & 1 & 2,22 & 2,94 & & 2,26 & - & - & - & - & - & - & - & - & - & - \\
\hline E. rostrifolia & 1 & 2,22 & 2,94 & 0,66 & 1,94 & 2 & 2,38 & 3,57 & 1,24 & 2,4 & - & - & - & - & _- \\
\hline E. uniflora & - & _- & - & - & - & 2 & 2,38 & 1,79 & 0,73 & 1,63 & 1 & 2,86 & 2,86 & 0,95 & 3,12 \\
\hline G. klotzschiana & - & - & - & - & - & 1 & 1,19 & 1,79 & 0,19 & 1,06 & - & - & - & - & - \\
\hline L. molleoides & 10 & 22,2 & & 18,5 & 18,47 & 2 & 2,38 & 3,57 & 1,62 & 2,52 & 2 & 5,71 & 5,71 & 28,8 & 13,36 \\
\hline L. divaricata & 2 & 4,44 & 5,88 & 5,18 & 5,17 & 4 & 4,76 & 3,57 & 15,5 & 7,97 & - & _ & _ & - & _ \\
\hline M. paraguariense & - & - & - & - & - & 1 & 1,19 & 1,79 & 1,76 & 1,58 & - & - & - & - & - \\
\hline M. elaeagnoides & - & - & _- & & - & 3 & 3,57 & 3,57 & 0,73 & 2,62 & 1 & 2,86 & 2,86 & 1,69 & 3,37 \\
\hline M. umbellata & - & - & - & - & - & 2 & 2,38 & 3,57 & 3,92 & 3,29 & - & - & _ & - & _ \\
\hline N. lanceolata & - & - & - & - & - & 1 & 1,19 & 1,79 & 0,67 & 1,22 & - & - & - & - & - \\
\hline N. megapotamica & 1 & 2,22 & 2,94 & 9,19 & 4,8 & 1 & 1,19 & 1,79 & 0,37 & 1,11 & - & - & - & - & - \\
\hline O. puberula & 10 & 22,2 & 20,6 & 39,2 & 27,37 & 6 & 7,14 & 5,36 & 8,98 & 7,17 & - & _ & _ & $\ldots$ & _ \\
\hline O. pulchella & - & - & - & - & _ & 1 & 1,19 & 1,79 & 1,01 & 1,33 & - & - & - & - & - \\
\hline P. sepium & - & - & - & - & - & 1 & 1,19 & 1,79 & 1,89 & 1,62 & - & - & - & - & - \\
\hline P. aduncum & - & - & - & 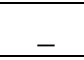 & - & - & - & - & - & - & 3 & 8,57 & 8,57 & 5,66 & 6,6 \\
\hline P. guajava & - & - & - & - & - & 1 & 1,19 & 1,79 & 0,75 & 1,24 & - & - & - & - & _- \\
\hline R. laxiflora & - & - & - & - & - & 2 & 2,38 & 3,57 & 0,87 & 2,27 & - & - & - & - & - \\
\hline S. bonplandii & _- & - & - & - & - & 2 & 2,38 & 1,79 & 1,26 & 1,81 & $\ldots$ & _- & _ & - & - \\
\hline S. romanzoffiana & 1 & 2,22 & 2,94 & 2,27 & 2,48 & - & - & - & - & - & - & - & - & - & - \\
\hline T. micranta & - & - & - & - & - & 1 & 1,19 & 1,79 & 3,43 & 2,14 & - & - & - & - & - \\
\hline T. clausseni & 1 & 2,22 & 2,94 & 0,25 & 1,81 & 3 & 3,57 & 1,79 & 1,95 & 2,44 & - & - & - & - & - \\
\hline Urera sp. & _ & _ & - & - & - & 3 & 3,57 & 3,57 & 2,37 & 3,17 & - & - & - & - & - \\
\hline Z. rhoifolium & - & - & - & - & - & 1 & 1,19 & 1,79 & 0,19 & 1,06 & 1 & 2,86 & 2,86 & 0,95 & 3,12 \\
\hline
\end{tabular}

$\mathrm{NI}=$ número de indivíduos; $\mathrm{DR}=$ densidade relativa; $\mathrm{FR}=$ frequência relativa; $\mathrm{DoR}=$ dominância relativa; $\mathrm{IVI}=$ índice de valor de importância $(\%)$. 
No estrato superior o índice de diversidade de Shannon (H') resultou em 2,29 e de equabilidade de Pielou (J') em 0,85, demonstrando alta uniformidade de distribuição das espécies presentes e baixa diversidade. Em relação à síndrome de dispersão, observou-se que a área amostrada apresentou composição de 86,6\% de espécies zoocóricas, 6,5\% anemocóricas e $6,5 \%$ autocóricas.

O índice de diversidade de Shannon (H') e de equabilidade de Pielou (J'), demonstraram maior uniformidade de distribuição das espécies presentes e baixa diversidade, comparado com os valores encontrados por Prado Júnior et al. (2012) que obteve, na mesma região, $\mathrm{H}^{\prime}=3,97$ e J' $=0,87$. O estrato médio resultou em 2,93 de diversidade no índice de Shannon e 0,87 de equabilidade de Pielou. De acordo com o Inventario Florestal Contínuo do Rio Grande do Sul, a diversidade das comunidades vegetais é média, com índice de Shannon de 2,47 (RIO GRANDE DO SUL, 2002).

Quanto a síndrome de dispersão dos frutos e sementes, o estrato médio apresentou $76,2 \%$ de dispersão zoocórica, 13,4\% autocórica e 10,4\% anemocórica. Quando há maior quantidade de espécies zoocóricas, esse fato pode ser considerado como indicativo de que essas comunidades vegetais estejam em estágios mais avançados de sucessão, pois a dispersão anemocórica ocorre mais em áreas de clareiras (PUIG, 2008), o que foi observado nesse estudo.

O índice de diversidade de Shannon para o estrato inferior médio resultou em 1,74 e de equabilidade de Pielou 0,73, sendo os menores valores encontrados entre os três estratos na área de estudo, demonstrando que este foi o que apresentou a dominância de uma espécie sobre as demais. Além disso, observou-se a presença de 17 indivíduos da espécie Actinostemon concolor, da família Euphorbiaceae, do total dos 35 identificados, o que pode ser justificado pela dispersão autocórica, a qual não permite que essa seja a longa distância da plantamãe (FIGUEIREDO, 2014).

A espécie Actinostemon concolor, com 20 indivíduos encontrados nas parcelas amostrais, obteve como Índice de Morisita o valor de 5,52, denotando padrão de distribuição agrupado. Esse padrão de distribuição expressa que os indivíduos ocorrem nos mesmos locais dentro da área em estudo, ou seja, estão agregados nas partes mais favoráveis do ambiente (ARRUDA; OMAR, 2007).

Casearia sylvestris apresentou o maior índice de valor de importância. Esta espécie é classificada como secundária inicial e ocorre em diversos tipos de solos úmidos ou secos, de textura arenosa ou argilosa, com drenagem boa a regular e fertilidade baixa a alta (CARVALHO, 2007).
A espécie Aiouea saligna, com sete indivíduos, obteve o valor de 1,43 no Índice de Morisita, apresentando também o padrão de distribuição agrupado.

As espécies Allophyllus edulis (19 indivíduos), Casearia sylvestris (26 indivíduos), Cupania vernalis (sete indivíduos), Litharea molleiodes (14 indivíduos), Luehea divaricata (seis indivíduos), Matayba elaeagnoides (quatro indivíduos) e Ocotea puberula (16 indivíduos) apresentaram como Índice de Morisita o valores de 1,$84 ; \quad 1,73 ; \quad 1,43 ; 2,31 ; 3,0 ; 2,5$ e 2,0 , respectivamente, sendo que essas espécies distribuíram-se também de forma agrupada.

A espécie Trichilia claussenii (quatro indivíduos) obteve o maior Índice de Morisita entre as dez espécies observadas, resultando no valor de 7,5, apresentando padrão de distribuição agrupado, assim como as demais. Essa espécie pertence ao grupo ecológico das secundárias tardias (VACCARO et al., 1999), sendo comum a sua ocorrência em fragmentos de Floresta Estacional Subtropical, como observado nesse estudo.

Verificou-se que as espécies apresentam em grande parte dispersão zoocórica, fato este que corrobora com pesquisas na Floresta Estacional Subtropical de Budke et al. (2005) e Sccoti et al. (2011), confirmando a importância dos agentes bióticos na manutenção de fluxo gênico em formações florestais.

\section{Conclusão}

Ocotea puberula, Lithraea molleoides, Casearia sylvestris, Allophylus edulis, Cupania vernalis, Luehea divaricata, Actinostemon concolor, Piper aduncum e Aiouea saligna foram as espécies de maior relevância da comunidade vegetal, apresentando valores de importância elevados.

As espécies mais representativas apresentaram padrão de distribuição espacial agrupado.

No estrato superior e médio o índice de diversidade de Shannon e equabilidade de Pielou apresentaram baixa diversidade e alta uniformidade de distribuição das espécies. No estrato inferior ocorreu a dominância de uma espécie sobre as demais.

Esse estudo possibilitou salientar tanto a importância ecológica das espécies em diferentes sinúsias, como também a dispersão dos frutos pela fauna, contribuindo para a regeneração natural e conservação da biodiversidade em fragmento de Floresta Estacional Subtropical. 


\section{Referências}

Arruda DM, Brandão DO, Costa FV, Tolentino GS, Brasil RD, Neto AS, Nunes YRF (2011) Structural aspects and floristic similarity among tropical dry forest fragments with different management histories in northern Minas Gerais, Brazil. Revista Árvore, 35(1): p. 131-142. DOI: 10.1590/S010067622011000100016

Arruda L, Omar D (2007) Florística e diversidade em um fragmento de Floresta Estacional Semidecidual Aluvial em Dourados, MS. Floresta, 37(2): p. 189-199. DOI: 10.5380/rf.v37i2.8649

Budke JC, Athayde EA, Giehl ELH, Záchia RA, Eisinger SM (2005) Composição florística e estratégias de dispersão de espécies lenhosas em uma floresta ribeirinha, arroio Passo das Tropas, Santa Maria, RS, Brasil. Iheringia. Série Botânica, 60(1): p. 17-24. DOI: ttps://isb.emnuvens.com.br/iheringia/article/view/2 02

Callegaro RM, Longhi SJ, Araujo ACB, Kanieski M R, Floss PA, Gracioli CR (2012) Estrutura do componente arbóreo de uma Floresta Estacional Decidual ripária em Jaguari, RS. Ciência Rural, 42(2): p. 305-3011. DOI: 10.1590/S010384782012000200019

Carvalho PER (2007) Circular Técnica 138: Cafezeiro-do-Mato Casearia sylvestris. 1 ed. Colombo: Embrapa. URL: https://www.infoteca.cnptia.embrapa.br/bitst ream/doc/313896/1/Circular138.pdf.

Chami LB, Araujo MM, Longhi SJ, Kielse P, Lúcio AD (2011) Mecanismos de regeneração natural em

Prado Júnior JÁ, Lopes SF, Schiavini I, Vale VS, Oliveira AP, Gusson AE, Neto OCD, Stein M (2012) Fitossociologia, caracterização sucessional e síndromes de dispersão da comunidade arbórea de remanescente urbano de Floresta Estacional Semidecidual em Monte Carmelo, Minas Gerais. Revista Rodriguésia, 63(3): p. 489-499, 2012. DOI: 10.1590/S2175-78602012000300001

Puig H (2008) A floresta tropical úmida. São Paulo: Editora UNESP Imprensa Oficial do Estado de São Paulo; França: Institut de Rechérche pour le Développement. 496 p.

Ribeiro MC, Metzger JP, Martensen AC, Ponzoni FJ, Hirota MM (2009) The Brazilian Atlantic Forest: How much is left, and how is the remaining forest distributed? Implications for conservation. diferentes ambientes de remanescente de Floresta Ombrófila Mista, São Francisco de Paula, RS. Ciência Rural,41(2): p. 251-259. DOI: 10.1590/S0103-84782011000200012

FAO - Organização das nações unidas para agricultura e alimentação (2010) Avaliação dos recursos florestais mundiais. URL: https:// www.fao.org.br/aif.asp.

Figueiredo MCS (2014) Florística da mata ciliar em São Martinho da Serra, RS, Brasil. Dissertação, Mestrado em Ciências Naturais e Exatas, Universidade Federal de Santa Maria, Santa Maria. $128 \mathrm{p}$.

Marcuzzo SB, Araújo MM, Longhi SJ (2013) Estrutura e relações ambientais de grupos florísticos em fragmento de floresta estacional subtropical. Revista Árvore, 37(2): p. 275-287. DOI: 10.1590/S0100-67622013000200009.

Matzenauer R, Radin B, Almeida IR de (2011) Atlas Climático: Rio Grande do Sul. Porto Alegre: Secretaria da Agricultura Pecuária e Agronegócio; Fundação Estadual de Pesquisa Agropecuária (FEPAGRO). 16 p.

Neto SS, Nakano O, Barbin D, Villa Nova NA (1976) Manual de ecologia dos insetos. Editora Ceres, Piracicaba, Brasil, 419 p.

Pinheiro F, Ribeiro JF (2001) Síndromes de dispersão de sementes em Matas de Galeria do Distrito Federal. In: Ribeiro JF, Fonseca CEL, Souza-Silva JC (eds.). Cerrado: caracterização e recuperação de Matas de Galeria. Embrapa Cerrados, Planaltina, v. 1, p. 335-378.

Biological conservation, 142(6): p. 1141-1153. DOI: 10.1016/j.biocon.2009.02.021

Rio Grande do Sul, Governo do Estado. Secretária Estadual do Meio Ambiente (2002). Inventário do Rio Grande do Sul. Porto Alegre: FATEC/SEMA. Disponível em:

http://w3.ufsm.br/ifcrs/frame.htm>.

Souza MR de, Ferreira MB, Sousa GG de, Alves AR, Holanda AC de (2020) Caracterização florística e fitossociológica do componente lenhoso de um fragmento florestal de caatinga em Serra do Mel, Rio Grande do Norte, Brasil. Nativa, 8(3): p. 329-335 DOI: 10.31413 /nativa.v8i3.9136

Sccoti MSV, Araujo MM, Wendler CF, Longhi SJ (2011) Natural regeneration mechanisms in a seasonal Deciduous Forest fragment. Ciência 
Quevedo et al.

Florestal, 21(3): p. $\quad$ 459-472. DOI: $10.5902 / 198050983803$

Shepherd GJ (2010) FITOPAC v. 2.1. Campinas, SP: Departamento de Botânica, Universidade Estadual

Scipioni MC, Longhi SJ, Reinert DJ, Araújo MM, Pedron F de A (2010) Distribuição do compartimento arbóreo em gradiente de relevo e solos na encosta Meridional da Serra Geral, RS. Ciência Rural, 40(6): p. 1295-1301. DOI: 10.1590/S0103-84782010005000090

Schilling AC, Batista JLF (2008) Curva de acumulação de espécies e suficiência amostral em florestas tropicais. Brazilian Journal of Botany, 31(1): p. 179-187. DOI: 10.1590/S010084042008000100016.

SEMA/RS, Secretaria Estadual do Meio Ambiente (2020) Unidades de Conservação, Porto Alegre. URL: http://www.sema.rs.gov.br.

de Campinas - UNICAMP.

Vaccaro S, Wojciechowski JC, Finger CAG (2011). Dinâmica do crescimento e produção em florestas secundárias em Santa Tereza. IN: Schumacher MV, Longhi SJ, Brun EJ, Kilca RV A floresta estacional subtropical caracterização e ecologia no rebordo do planalto meridional. Santa Maria: Editora Pallotti. p. 171-194.

Vaccaro S, Longhi SJ, Brena DA (1999) Aspectos da composição florística e categorias sucessionais do estrato arbóreo de três subseres de uma Floresta Estacional Decidual, no Município de Santa Tereza - RS. Ciência Florestal, 9(1): p. 1-18. 